\title{
artigo
}

Vasconcelos, A.A.; Medeiros, K.S.; Perez, A.S.; Fernandes, D.M.A.P.

Estamos ofertando consultas de pré-natal humanizadas na Atenção Básica?

\section{Estamos ofertando consultas de pré-natal humanizadas na Atenção Básica?}

\author{
Are we offering humanized prenatal consultations in Primary Care?
}

¿Ofrecemos consultas prenatales humanizadas en Atención Primaria?

\begin{abstract}
RESUMO
Objetivo: analisar a produção científica sobre a importância da realização e acompanhamento adequado do pré-natal na atenção básica. Métodos: revisão integrativa nas bases de dados LILACS e SciELO, utilizando os descritores "cuidado pré-natal" e "Atenção primária à saúde". Foram analisados 281 títulos e selecionados 7 artigos. Resultados: A atenção básica ainda force assistência pré-natal de baixa qualidade e não humanizada. Realizar e acompanhar adequadamente o pré-natal resulta em benefícios para a gestante, para o binômio mãe-filho e também para a equipe prestadora do cuidado. Envolve a atuação multiprofissional. E, os estudos demonstram que a atuação conjunta de médicos e enfermeiros aumenta o percentual de orientações preconizadas. Conclusão: 0 conhecimento dos profissionais da saúde deve ser ampliado quanto às técnicas e habilidades a serem compartilhadas com a gestante para facilitar a fluidez do serviço de saúde, beneficiando ambas as partes, bem como o apoio da gestão.
\end{abstract}

DESCRITORES: Atenção primária à Saúde; Cuidado pré-natal; Gestantes.

\section{ABSTRACT}

Objective: to analyze the scientific production on the importance of carrying out and adequate monitoring of prenatal care in primary care. Methods: integrative review in the LILACS and SciELO databases, using the keywords "prenatal care" and "Primary health care". 281 titles were analyzed and 7 articles were selected. Results: Primary Care still forces low quality and non-humanized prenatal care for most pregnant women. Performing and properly monitoring prenatal results in benefits for the pregnant woman, for the mother-child binomial and also for the care team. It involves multiprofessional action. And, studies show that the joint action of doctors and nurses increases the percentage of recommended guidelines. Conclusion: The knowledge of health professionals should be expanded as to the techniques and skills to be shared with the pregnant woman to facilitate the fluidity of the health service, benefiting both parties, as well as management support.

DESCRIPTORS: Primary Health Care; Prenatal care; Pregnant women.

\section{RESUMEN}

Objetivo: analizarla producción científica sobre la importancia de la realización y seguimiento adecuado de la atención prenatal en atención primaria. Métodos: revisión integradora en las bases de datos LILACS y SciELO, utilizando las palabras clave "atención prenatal" y "Atención primaria de salud". Se analizaron 281 títulos y se seleccionaron 7 artículos. Resultados: La Atención Primaria todavía obliga a la atención prenatal de baja calidad y no humanizada para la mayoría de las mujeres embarazadas. Realizar y monitorear adecuadamente los resultados prenatales en beneficios para la gestante, para el binomio madre-hijo y también para el equipo de atención. Implica una acción multiprofesional. Y, los estudios muestran que la acción conjunta de médicos y enfermeras aumenta el porcentaje de pautas recomendadas. Conclusión: Se debe ampliar El conocimiento de los profesionales de La salud en cuanto a las técnicas y habilidades a compartir con la gestante para facilitar la fluidez Del servicio de salud, beneficiando a ambas partes, así como el apoyo a la gestión.

DESCRIPTORES: Atención Primaria de Salud; atención prenatal; Mujeres embarazadas.

RECEBIDO EM: 06/02/2021 APROVADO EM: 22/02/2021

\section{Andressa Andrade de Vasconcelos}

Graduada na Faculdade de Medicina Nova Esperança; residente do programa de Residência Médica em Medicina de Família e Comunidade da Secretaria Municipal de Saúde de João Pessoa em parceria com a Faculdade de Ciências Médicas da Paraíba (FCM/PB-AFYA).

ORCID: 0000-0002-7820-3110 


\section{Karina Suassuna de Medeiros}

Graduada na Faculdade de Medicina Nova Esperança; residente do programa de Residência Médica em Medicina de Família e Comunidade da Secretaria Municipal de Saúde de João Pessoa em parceria com a Faculdade de Ciências Médicas da Paraíba (FCM/ PB-AFYA).

ORCID: 0000-0002-1086-115X

\section{Alejandra Soledad Perez}

Graduada em Medicina pela Escuela Latinoamericana de Medicina, residente de Medicina de Família e Comunidade da Secretaria Municipal de Saúde de João Pessoa em parceria com a Faculdade de Ciências Médicas da Paraíba (FCM/ PB-AFYA).

ORCID: 0000-0002-0608-9101

\section{Denise Mota Araripe Pereira Fernandes}

Preceptora e docente do Programa de Residência Médica em Medicina de Família e Comunidade da Secretaria Municipal de Saúde de João Pessoa em parceria com a Faculdade de Ciências Médicas da Paraíba (FCM/PB-AFYA).

ORCID: 0000-0001-7804-882X

\section{INTRODUÇÃO}

0 pré-natal tem como objetivo cuidar do desenvolvimento da gestação, possibilitando parto de um recém-nascido saudável, minorando impactos a saúde materna. Deve incluir abordagens psicossociais, atividades educativas e preventivas ${ }^{(1)}$. Inclui ações de proteção e promoção à saúde, detecção e tratamento precoce de agravos que podem ocorrer durante a gravidez e até 42 diasapós oparto ${ }^{(2)}$.

No Sistema Único de Saúde (SUS) o pré-natal de baixo risco deve ser realizado na Atenção Básica $(A B)$, a principal porta de entrada para o acompanhamento da gestante. $\mathrm{A} A \mathrm{~B}$ configura-se por um grupo de ações em saúde, voltadas tanto para indivíduos como para coletividades, abrangendo dentre seus instrumentos a promoção e a proteção em saúde ${ }^{(3,4)}$. Destaca-se que a Política Nacional de Atenção Básica (PNAB) considera equivalentes os termos $\mathrm{AB}$ e Atenção Primaria à Saúde (APS) ${ }^{(5)}$.

Em 2000, o Ministério da Saúde (MS) instituiu o Programa de Humanização do Pré-Natal e Nascimento (PHPN) para promover a articulação dos serviços de saúde durante o parto e o pré-natal, normatizando as ações de saúde ${ }^{(6)}$. Em 2011, por meio da Portaria 1459, foi instituída a Rede Cegonha, com o objetivo de proporcionar atendimento humanizado à gestante, garantindo acesso ao pré-natal de qualidade, acolhimento com avaliação e classificação de risco e vulnerabilidade, como também vinculação da gestante à unidade de referência, transporte e segurança na atenção ao parto ${ }^{(7)}$.

A PHPN recomenda alguns exames e no mínimoseis consultas de pré-natal, sendo a primeira antes do quarto mês e uma no puerpério(7). Em um estudo realizado para comparar a qualidade da assistência pré-natal às recomendações do PHPN, observou-se que a assistência oferecida às gestantes do SUS é inadequada visto início tardio da assistência, insuficiente número de consultas, cobertura insuficiente dos testes padronizados e poucas orientações ${ }^{(8)}$.

É imperativo que o acesso precoce a cuidados pré-natais de qualidade de fato aconteça na $\mathrm{AB}$, visto promover intervenção oportuna, estratificação de risco e oferta de orientações de fácil acesso. O objetivo do estudo foi revisitar a literatura em busca de evidências que retifiquem a importância do pré-natal na $\mathrm{AB}$ para fortalecer os processos de cuidado.

\section{MÉTODO}

Trata-se de pesquisa descritiva, exploratória, retrospectiva a partir de revisão integrativa na literatura científica. Dividida em 6 etapas: $1^{\text {a }}$ ) identificação do tema e seleção da questão de pesquisa, $2^{a}$ ) Determinação de critérios de inclusão e exclusão de estudos ou busca na literatura, $3^{\mathrm{a}}$ ) Escolha das informações a serem retiradas, categorização dos estudos, $4^{a}$ ) Análise das informações ou estudos incluídos na revisão integrativa, $5^{\text {a }}$ ) interpretação dos resultados obtidos e 6a) Apresentação da revisão/síntese do conhecimento.

$\mathrm{Na}$ primeira etapa elaborou-se a pergunta de pesquisa: porque é importante realizar pré-natal de qualidade na $\mathrm{AB}$ ?

Já para a segunda etapa, ao delimitar os critérios de inclusão e exclusão dos estudos. Definiu-se como critérios de inclusão: publicações em formato de artigo, em periódicos nacionais e internacionais, em português e inglês, publicados no período de 2016 a 2021, com textos completos e disponíveis, que apresentem resultados relevantes e que respondam à pergunta de pesquisa. Como critérios de exclusão foram artigos publicados fora do período de busca, que não atendem aos objetivos da pesquisa e que não estavam disponíveis na íntegra.

A busca foi feita de novembro e dezembro de 2020, nas bases de dados: Literatura Latino-americana e do Caribe em ciências da saúde (LILACS) e Base de dados Scientific Electronic Library online (SciELO). Utilizou-se os descritores em Ciências da Saúde (DeCS) da Biblioteca Virtual de Saúde e os descritores em língua inglesa Medical Subject Headings (MeSH): Cuidado pré-natal and Atenção Primária à saúde.

A terceira etapa apoiou-se na definição das informações dos estudos selecionados, que foram categorizados em um quadro formulado pelo pesquisador com as informações de identificação do periódico selecionado. A análise das informações, contempladas na quarta etapa, a partir dos artigos tabulados, foram descritas por meio 


\section{artigo}

Vasconcelos, A.A.; Medeiros, K.S.; Perez, A.S.; Fernandes, D.M.A.P.

Estamos ofertando consultas de pré-natal humanizadas na Atenção Básica?

de análise crítica, as semelhanças e diferenças entre os estudos. Os resultados foram interpretados na quinta etapa, avaliando a importância de realizar pré-natal de qualidade na $A B$, considerando as evidências científicas mais importantes sobre o assunto. $\mathrm{Na}$ última e sexta etapa, construiu-se uma síntese, em forma de resumo sobre tais evidências encontradas, que identificaram de forma clara e relevante o real impacto do pré-natal de qualidade na $A B$.

\section{RESULTADOS}

Foram identificados 281 artigos. Destes, foram excluídos 163 artigos por não atenderem aos critérios de inclusão, 104 após a leitura dos títulos e 7 pelo resumo, sendo selecionados 7 artigos para compor o estudo.

Os artigos escolhidos estão apresentados no Quadro 1, de acordo com o "Base de dados", "país de origem", o "Título" do estudo; o "tipo de estudo", os "autores" e "periódicos e ano de publicação".

\section{DISCUSSÃO}

As ações desenvolvidas no atendimento pré-natal que promovem diálogo entre a gestante, familiares e profissional de saúde facilitam a execução do plano de parto ${ }^{(11)}$. $E$ na $\mathrm{AB}$ que os vínculos entre profissionais e usuários, bem como a participação da família, são favorecidos, criando-se uma relação de confiança e escuta ativa, o que gera respeito e valorização da gestante, melhorando a assistência ${ }^{(16)}$.

O cuidado humanizado torna possível a aproximação adequada entre o profissional e a gestante de modo a atender à complexidade do período gravídico. A humanização é peça fundamental no período pré-natal, pois uma pessoa só se liga a outra se houver empenho na relação, ou seja, investimento em açôes que tenham como foco o bem-estar da pessoa grávida, tornando a relação mais efetiva e proveitosa e centrada na paciente ${ }^{(13)}$.

Além disso, o acompanhamento pré-natal por uma equipe multiprofissional é extremamente importante ${ }^{(10)}$. As consultas quando realizadas em equipe apresentam maiores chances de orientaçôes humanizadas, conforme demonstra o estudo de Marques et al. ${ }^{(9)}$ : consultas realizadas por enfermeiros e médicos apresentaram $41 \%$ de chance de maior adequação às orientações, comparado com exclusivo médico. $\mathrm{O}$
MS recomenda abordagem dos seguintes temas: importância do pré-natal; higiene oral da mãe e da criança; atividade física adequada; alimentação saudável; uso de medicamentos, álcool e tabaco; desenvolvimento da gestação; modificações corporais e emocionai; medos e fantasias com relação à gestação e parto; sinais comuns na gestação; orientações nas queixas frequentes; importância da participação paterna; parto e desenvolvimento do vínculo entre pai e filho; cuidados pós-parto, com recém nascido (exemplo: shantala para bebês) e estimulo ao retorno dos serviços de saúde ${ }^{(2)}$.

Ações de educação em saúde contribuem para a promoção da saúde. No relato de experiência de Silva et al. ${ }^{(17)}$, as rodas de conversa foram realizadas por enfermeiros, psicólogos, fisioterapeutas, odontólogos, assistentes sociais e biólogo com especialização em fisiologia e embriologia, demonstrando a importância da participação da equipe multidisciplinar para à formação de opiniões e a consolidação do conhecimento sobre gestação e partos saudáveis e bem assistidos.

O MS recomenda para a saúde da mulher: assistência pré-natal, perinatal, puerperal e dorecém-nascido na $\mathrm{AB}$ em equipe, uma vez que

Quadro 1: Características gerais dos artigos selecionados

\begin{tabular}{|c|c|c|c|c|c|}
\hline $\begin{array}{l}\text { BASE DE } \\
\text { DADOS }\end{array}$ & $\begin{array}{l}\text { PAÍS DE } \\
\text { ORIGEM }\end{array}$ & TítULo & TIPO DE ESTUDO & AUTORES & $\begin{array}{l}\text { PERIÓDICOS E ANO DE } \\
\text { PUBLICAÇÃO }\end{array}$ \\
\hline SciELO & Brasil & $\begin{array}{l}\text { Orientações às gestantes no pré-natal: a im- } \\
\text { portância do cuidado compartilhado na atenção } \\
\text { primária em saúde }\end{array}$ & $\begin{array}{l}\text { Quantitativo, } \\
\text { transversal }\end{array}$ & $\begin{array}{l}\text { Marques et } \\
\quad \text { al. }^{(9)}\end{array}$ & Escola Anna Nery (2021) \\
\hline LILACS & Brasil & $\begin{array}{l}\text { Assistência pré-natal segundo registros } \\
\text { profissionais presentes na caderneta da gestante }\end{array}$ & $\begin{array}{l}\text { Quantitativo, } \\
\text { descritivo }\end{array}$ & $\begin{array}{l}\text { Castro et } \\
\text { al.(10) }\end{array}$ & $\begin{array}{l}\text { Revista enfermagem UFSM } \\
\text { (2020) }\end{array}$ \\
\hline LILACS & Brasil & $\begin{array}{c}\text { Pré-Natal Como Facilitador Na Participação Do } \\
\text { Acompanhante No Processo De Trabalho De } \\
\text { Parto E Parto }\end{array}$ & $\begin{array}{l}\text { Qualitativo, descri- } \\
\text { tivo, exploratório }\end{array}$ & $\begin{array}{l}\text { Souza et } \\
\text { al. }^{(11)}\end{array}$ & $\begin{array}{l}\text { Revista de pesquisa: cuida- } \\
\text { do é Fundamental (2020) }\end{array}$ \\
\hline $\begin{array}{l}\text { SciELO } \\
\text { LILACS }\end{array}$ & Brasil & $\begin{array}{l}\text { Não realização de teste sorológico para sífilis du- } \\
\text { rante o pré-natal: prevalência e fatores associados }\end{array}$ & $\begin{array}{l}\text { Quantitativo, } \\
\text { transversal }\end{array}$ & $\begin{array}{l}\text { Cesar et } \\
{\text { al. } .^{(12)}}\end{array}$ & $\begin{array}{l}\text { Revista brasileira epide- } \\
\text { miologia (2020) }\end{array}$ \\
\hline LILACS & Brasil & O cuidado no pré-natal: um valor em questão. & $\begin{array}{l}\text { Qualitativo, descri- } \\
\text { tivo, exploratório }\end{array}$ & $\begin{array}{l}\text { Silva et } \\
\text { al. }{ }^{(13)}\end{array}$ & $\begin{array}{l}\text { Cogitare enfermagem } \\
\text { (2017) }\end{array}$ \\
\hline LILACS & Brasil & $\begin{array}{l}\text { Avaliação das consultas de pré-natal: adesão do } \\
\text { pré-natal e complicações na saúde materno infantil. }\end{array}$ & $\begin{array}{l}\text { Quantitativo, } \\
\text { avaliativo }\end{array}$ & $\begin{array}{l}\text { Pereira et } \\
\text { al. }{ }^{(14)}\end{array}$ & $\begin{array}{l}\text { Revista Ciência Plural } \\
\text { (2017) }\end{array}$ \\
\hline SciELO & Brasil & $\begin{array}{l}\text { Atenção pré-natal e fatores de risco associados } \\
\text { à prematuridade e baixo peso ao nascer em } \\
\text { capital do nordeste brasileiro }\end{array}$ & Caso-controle & $\begin{array}{l}\text { Gonzaga et } \\
\text { al. }{ }^{(15)}\end{array}$ & $\begin{array}{l}\text { Ciência saúde coletiva } \\
\qquad(2016)\end{array}$ \\
\hline
\end{tabular}


esses profissionais somam contribuições para prestar assistência de qualidade ${ }^{(1)}$.

Vale ressaltar, que as orientações durante o acompanhamento pré-natal, são práticas profissionais que não implicam custos financeiros adicionais, mas dependem do protagonismo e atitudes dos profissionais ${ }^{(9)}$. No estudo de Castro et al. ${ }^{(10)}$, $80,8 \%$ das mulheres foram atendidas tanto por médicos como por enfermeiros queintercalam as consultas em busca de um atendimento interdisciplinar. Outro estudo, realizado por Costa et al. ${ }^{(18)}$, em Goiânia, encontraram valores inferiores, com $45,4 \%$ das consultas forma realizadas por médicos e enfermeiros e $53,6 \%$ foram atendidas, apenas, pelo médico.

É importante que os profissionais realizem o registro de altura, peso, índice de massa corpórea (IMC) pré-gravídico, batimentos cardíaco fetais e aferição da pressão arterial, atualizados a cada consulta. Alterações no IMC devem ser estimuladas ao diálogo sobre cuidado, para evitar modificações na glicemia e pressão arterial da gestante, bem como a preservação da saúde do feto ${ }^{(10)}$.

Pereira et al. ${ }^{(14)}$, ao avaliarem a correlação entre adesão ao pré-natal e complicações perceberam que $86 \%$ das mães que aderiram ao pré-natal, não complicaram, ao passo que as $13 \%$ menos participativas tiveram complicações, tais como: sangramento $(9,4 \%)$, convulsão $(3,1 \%)$, infecção urinária $(1,6 \%)$, diabetes (1\%) e hipertensão arterial $(8,4 \%)$.

Verificar a pressão nas consultasé indispensável para avaliar os riscos relacionados à hipertensão arterial, pois prevenir e detectar precocemente ajuda a adotar condutas que evitam complicações ${ }^{(19)}$. Os distúrbios hipertensivos da gestação são uma das principais causas de mortalidade materna e perinatal em todo o mundo, com prevalência de hipertensão crônica em $0,9-1,5 \%$ das grávidas, sendo a pré-eclâmpsia a principal complicação, com prevalência global de 2 a $8 \%$ das gestações globalmente. O Brasil, prevê uma incidência de $1,5 \%$ para pré-eclâmpsia e de 0,6\% para eclâmpsia ${ }^{(20)}$. Em caso de tais alterações, a gestante éencaminhada para atenção especializada ${ }^{(10)}$.

Pouco adianta realizar os procedimen-

tos, se os mesmos não forem registrados. Falhas de registro na caderneta da gestante influenciam o cuidado do binômio mãe-filho no parto e puerpério. Castro et al. ${ }^{(10)}$, encontraram altos índices de falhas, principalmente quanto aos exames laboratoriais e avaliação nutricional. No estudo de Gonzaga et al. ${ }^{(15)}$ as mães que apresentaram inadequação da atenção pré-natal em relação à realização da rotina de exames laboratoriais básicos ( $76 \%$ dos casos), tiveram chance aumentada para a ocorrência da prematuridade e/ou baixo peso ao nascer.

É importante que os profissionais realizem o registro de altura, peso, índice de massa corpórea (IMC) pré-gravídico, batimentos cardíaco fetais e aferição da pressão arterial, atualizados a cada consulta.

O MS preconiza a solicitação: ABO-Rh, hemoglobina/hematócrito, glicemia de jejum, VDRL, urina tipo I, testagem anti-HIV, hepatite $\mathrm{B}$, citomegalovírus, rubéola e toxoplasmose ${ }^{(2)}$.

Outro quesito abordado nos estudos selecionados relaciona-se ao diagnóstico adequado da sífilis na gravidez para evitar sífilis congênita. Cesar et al. ${ }^{(12)}$, propõem medidas para aumentar a cobertura do teste soroló- gico: a disponibilização dos teste rápido nas unidades de saúde; realização de campanhas, incentivo a solicitação do exame na consulta de pré-natal; priorização dos pacientes com perfil de poucas consultas. O MS preconiza que o rastreamento da sífilis durante o pré-natal deve ser realizado pelo menos mínimo duas vezes na gestação (início do pré-natal e próximo à $30^{\mathrm{a}}$ semana) e no momento da internação hospitalar ${ }^{(2)}$.

O pré-natal, no Brasil, apresenta iniquidades e baixa qualidade no atendimento, principalmente nas regiões pobres ${ }^{(21)}$, bem como inadequação da infra-estrutura da rede de $A B$ que realiza o pré-natal, baixa adequação de ações clínicas com baixa capacidade de gestão de equipes ${ }^{(22)}$. No estudo de Carvalho et al. ${ }^{(23)}$ o cuidado pré-natal foi considerado inadequado para $89,1 \%$ das mulheres.

Os principais problemas relacionados a adequação ao PHPN por Mendes et al. ${ }^{(24)}$ foram início tardio do pré-natal; número insuficiente de consultas; poucas orientações, inclusive sobre a maternidade de referência para o parto; peregrinação no anteparto; falta de priorização das gestantes de maior risco e problemas na articulação com outros serviços de saúde materno-infantil.

Portanto para a redução da morbimortalidade materno-infantil, bem como de complicações, é imprescindível o início seja precoce do acompanhamento e cumprimento das recomendações do MS, quanto as ações de promoção, prevenção, diagnóstico e tratamento dos problemas que podem surgir na gravidez ${ }^{(24)}$. Além de ser integral e equânime, fortalecendo as redes regionais voltadas para a inclusão social ${ }^{(25)}$.

\section{CONCLUSÃO}

Vinte e um anos após a PHPN a AB ainda force assistência pré-natal de baixa qualidade e não humanizada para grande parte das gestantes. Acompanhar adequadamente o pré-natal resulta em benefícios para a mulher, para o binômio mãe-filho e reduz iniquidades. Envolve a atuação multiprofissional e tem na atuação conjunta de médicos e enfermeiros resultados mais robustos e maiores chances de seguimento das orientações preconizadas. O início tardio do pré- 


\section{artigo}

Vasconcelos, A.A.; Medeiros, K.S.; Perez, A.S.; Fernandes, D.M.A.P.

Estamos ofertando consultas de pré-natal humanizadas na Atenção Básica?

-natal aumenta os riscos de complicações a mãe e bebê e ainda é realidade na $\mathrm{AB}$.

A revisão integrativa não esgota as necessidades sobre revisitação do tema, porém alerta sobre a potência da partilha multiprofissional, desfalque na assistência e necessidade de ampliar as técnicas e habilidades a serem compartilhadas com a gestante para facilitar a fluidez do entendimento, evitando riscos e não imputando custos, logo beneficiando pessoas, profissionais e o sistema de saúde.

\section{REFERÊNCIAS}

1. Brasil. Ministério da Saúde. Secretaria de Atenção à Saúde. Departamento de Atenção Básica. Atenção ao pré-natal de baixo risco. Brasília: Ministério da Saúde; 2013.

2. Brasil. Ministério da Saúde. Secretaria de Atenção à Saúde. Departamento de Ações Programáticas Estratégicas. Área Técnica de Saúde da Mulher. Pré-natal e Puerpério: atenção qualificada e humanizada - manual técnico/Ministério da Saúde, Secretaria de Atenção à Saúde, Departamento de Ações Programáticas Estratégicas - Brasília: Ministério da Saúde, 2005. 158 p.

3. Brasil. Ministério da Saúde. Portaria n².488, de 21 de outubro de 2011. Aprova a Política Nacional de Atenção Básica, estabelecendo a revisão de diretrizes e normas para a organização da Atenção Básica, para a Estratégia Saúde da Família (ESF) e o Programa de Agentes Comunitários de Saúde (PACS). Diário Oficial da União. Brasília. 2011.

4. Brasil. Ministério da Saúde. Portaria n² 2.436, de 21 de setembro de 2017. Aprova a Política Nacional de Atenção Básica, estabelecendo a revisão de diretrizes para a organização da Atenção Básica, no âmbito do Sistema Único de Saúde (SUS). Diário Oficial da União. Brasília. 2017.

5. Brasil. Ministério da Saúde. Portaria n².446, de 11 de novembro de 2014. Redefine a Política Nacional de Promoção da Saúde (PNPS). Diário Oficial da União. Brasília. 2014

6. Brasil. Ministério da Saúde. Portaria GM/MS n 569. Institui o Programa de Humanização no Pré-natal e Nascimento, no âmbito do Sistema Único de Saúde. 2000.

7. Brasil. Ministério da Saúde. Portaria n 1.459, de 24 de junho de 2011. Institui no âmbito do Sistema Único de Saúde SUS a Rede Cegonha. Diário Oficial da União. 2011.

8. Dantas DS, Mendes RB, Santos JMJ, Valença TS, Mahl C, Barreiro MSC. Quality of prenatal care in the Unified Health System. Rev Enferm UFPE online. 2018; 12(5):1365-71.

9. Marques BL, Tomasi YT, Saraiva SS, Boing AF, Geremia DS. Orientações às gestantes no pré-natal: a importância do cuidado compartilhado na atenção primária em saúde. Esc. Anna Nery. 2021;25(1):e20200098.

10. Castro LLS, Oliveira IG, Bezerra RA, Sousa LB, Anjos SJSB, Santos LVF. Assistência pré-natal segundo registros profissionais presentes na caderneta da gestante. Rev. Enferm. UFSM REUFSM. 2020;10(16):1-18.

11. Souza MAR, Wall ML, Thuler ACMC, Souza SRRK. Pré-natal como facilitador na participação do acompanhante no processo de trabalho de parto e parto. Rev. Pesqui. 2020;12: 196-201.

12. Cesar JA, Camerini AV, Paulitsch RG, Terlan RJ. Não realização de teste sorológico para sífilis durante o pré-natal: prevalência e fatores associados. Rev Bras Epidemiol. 2020; 23: e200012.
13. Silva LA, Alves VH, Rodrigues DP, Santos MV, Guerra JVV Marchiori GRS. O cuidado no pré-natal: um valor em questão. Cogitare enferm. 2017;22(2): 01-08.

14. Pereira DO, Ferreira TLS, Araújo DV, Melo KDF, Andrade FB. Avaliação das consultas de pré-natal: adesão do pré- natal e complicações na saúde materno-infantil. Rev. Ciênc. Plur. 2017;3(3): 2-15.

15. Gonzaga ICA, Santos SLD, Silva ARV, Campelo V. Atenção pré-natal e fatores de risco associados à prematuridade e baixo peso ao nascer em capital do nordeste brasileiro. Ciência \& Saúde Coletiva. 2016; 21(6):1965-1974.

16. Silva MZN, Andrade AB, Bosi MLG. Acesso e acolhimento no cuidado pré-natal à luz de experiências de gestantes na Atenção Básica. Saúde Debate. 2014;38(103): 805-816

17. Silva IS, Xavier PB, Araujo TLL, Paulino DA, Queiroz CG, Brandão GCG. As rodas de conversa como ferramenta complementar ao pré-natal. Extensão em Ação. 2020;19(1):84-93.

18. Costa CSC, Vila VSC, Rodrigues FM, Martins CA, Pinho LMO. Características do atendimento pré-natal na Rede Básica de Saúde. Rev Eletrônica Enferm. 2013 ;15(2):516-22.

19. Lima LFC, Davim RMB, Silva RAR, Costa DARS, Mendonça AEO.. Importância do exame físico da gestante na consulta do enfermeiro. Rev enferm UFPE online. 2014;8(6):1502-9.

20. Barroso WKS, Rodrigues CIS, Bortolotto LA, Gomes MAM, Brandão AA, Feitosa ADM, Machado CA, et al. Diretrizes Brasileiras de Hipertensão Arterial - 2020. 2020;00(00):00.

21. Mario DN, Rigo L, Boclin KLS, Malvestio LMM, Anziliero D, Horta BL et al . Qualidade do Pré-Natal no Brasil: Pesquisa Nacional de Saúde 2013. Ciênc. saúde coletiva. 2019;24(3):12231232.

22. Guimarães WSG, Parente RCP, Guimarães TLF, Garnelo L. Acesso e qualidade da atenção pré-natal na Estratégia Saúde da Família: infraestrutura, cuidado e gestão. Cad. Saúde Pública.2018;34(5):e00110417.

23. Carvalho RAS, Santos VS, Melo CM, Gurgel RQ, Oliveira CCC. Avaliação da adequação do cuidado pré-natal segundo a renda familiar em Aracaju, 2011. Epidemiol. Serv. Saúde. 2016; 25(2): 271-280.

24. Mendes RB, Santos JMJ, Prado DS, Gurgel RQ, Bezerra FD, Gurgel RQ. Avaliação da qualidade do pré-natal a partir das recomendações do Programa de Humanização no Pré-natal e Nascimento. Ciênc. saúde coletiva. 2020; 25(3):793-804.

25. Cunha AC, Lacerda JT, Alcauza MTR, Natal S. Avaliação da atenção ao pré-natal na Atenção Básica no Brasil. Rev. Bras. Saúde Mater. Infant. 2019;19(2):447-458. 